\title{
Layered nanoemulsions as mucoadhesive buccal systems for controlled delivery of oral cancer therapeutics
}

This article was published in the following Dove Press journal:

International Journal of Nanomedicine

23 February 2015

Number of times this article has been viewed

\author{
Amy Gavin' \\ Jimmy TH Pham² \\ Dawei Wang ${ }^{2}$ \\ Bill Brownlow ${ }^{3}$ \\ Tamer A Elbayoumi ${ }^{3}$ \\ 'College of Dental Medicine, ${ }^{2}$ Arizona \\ College of Osteopathic Medicine, \\ ${ }^{3}$ Department of Pharmaceutical \\ Sciences, College of Pharmacy- \\ Glendale, Midwestern University, \\ Glendale, AZ, USA
}

\begin{abstract}
Oral cavity and oropharyngeal cancers are considered the eighth most common cancer worldwide, with relatively poor prognosis ( $62 \%$ of patients surviving 5 years, after diagnosis). The aim of this study was to develop a proof-of-concept mucoadhesive lozenge/buccal tablet, as a potential platform for direct sustained delivery of therapeutic antimitotic nanomedicines. Our system would serve as an adjuvant therapy for oral cancer patients undergoing fullscale diagnostic and operative treatment plans. We utilized lipid-based nanocarriers, namely nanoemulsions (NEs), containing mixed-polyethoxylated emulsifiers and a tocopheryl moietyenriched oil phase. Prototype NEs, loaded with the proapoptotic lipophilic drug genistein (Gen), were further processed into buccal tablet formulations. The chitosan polyelectrolyte solution overcoat rendered NE droplets cationic, by acting as a mucoadhesive interfacial NE layer. With approximate size of $110 \mathrm{~nm}$, the positively charged chitosan-layered $\mathrm{NE}(+25 \mathrm{mV})$ vs negatively charged chitosan-free/primary aqueous NE $(-28 \mathrm{mV})$ exhibited a controlled-release profile and effective mucoadhesion for liquid oral spray prototypes. When punch-pressed, porous NEbased buccal tablets were physically evaluated for hardness, friability, and swelling in addition to ex vivo tissue mucoadhesion force and retention time measurements. Chitosan-containing $\mathrm{NE}$ tablets were found equivalent to primary NE and placebo tablets in compression tests, yet significantly superior in all ex vivo adhesion and in vitro release assays $(P \leq 0.05)$. Following biocompatibility screening of prototype chitosan-layered NEs, substantial anticancer activity of selected cationic Gen-loaded NE formulations, against two oropahryngeal carcinomas, was observed. The data strongly indicate the potential of such nanomucoadhesive systems as maintenance therapy for oral cancer patients awaiting surgical removal, or postresection of identified cancerous lesions.
\end{abstract}

Keywords: isoflavone, genistein, chitosan, squamous cell carcinomas

\section{Introduction}

Oral cancer, as a subtype of head and neck cancer, is any cancerous tissue growth located in the oral cavity or the oropharynx. There are several types of oral cancers, of which approximately $90 \%$ are squamous cell carcinomas, originating in the tissues lining the mouth, tongue, and lips. Patients with head and neck cancers have been recognized to have an increased risk of developing a second primary tumor of the upper aerodigestive tract. ${ }^{1}$ Oral squamous cell carcinomas, regardless of various histological features, predominantly carry a high risk of metastatic spread to other body organs. ${ }^{1}$

While early cancers (stage I and stage II) of the lip and oral cavity are highly curable by surgery or radiation therapy, ${ }^{2}$ the presence of a positive margin or a tumor depth $>5 \mathrm{~mm}$ significantly increases the risk of local recurrence and often calls for aggressive combined modality treatment. ${ }^{3}$ Advanced cancers (stage III and stage IV) of 
the lip and oral cavity represent a wide spectrum of challenges for both the surgeon and radiation oncologist, mostly because local recurrence, regional, and/or distant metastases are quite common in this patient population. ${ }^{4}$

In recent years, the development of controlled drug delivery systems to, or through, the oral mucosa via the application of bioadhesive polymers, has gained significant attention. The buccal and sublingual routes are becoming the most commonly used ones for local or systemic delivery. ${ }^{5}$ Specifically, the oral cavity appears to offer the unique advantages of facile accessibility, a robust yet relatively permeable epithelial barrier for drug transport, unidirectional drug flux, quick and easy removal of the dosage form upon request, good drug absorption, and bypass of hepatic firstpass metabolism, thus collectively leading to enhancement of bioavailability, as well as patient compliance. ${ }^{6}$

Different dosage form designs, such as adhesive gels, tablets, films, patches, ointments/pastes, and mouthwashes/ sprays, have exploited the buccal route for both local and systemic drug delivery. ${ }^{5,7}$ Locally, mucoadhesive dosage forms are used to treat pathological conditions such as aphthous ulceration gingivitis, periodontal diseases, and xerostomia. For their remarkable flexibility and comfort in application, mucoadhesive tablets have been the most commonly used dosage form for buccal drug delivery as they can be applied to different regions within the oral cavity, such as cheeks, lips, gums, and palate. ${ }^{8}$ In addition, mucoadhesive tablets can overcome the relatively short residence time of oral gels/solutions, which are easily washed off by mouth saliva and/or food. ${ }^{7,9}$

However, mucosal delivery of active compounds by the buccal route remains quite challenging. Two important parameters are necessary to be considered while developing any mucoadhesive oral tablet. First, it is crucial to prolong the time of contact between the drug formulation and the oral mucosal membrane, especially for controlled-release dosage forms. ${ }^{10}$ Second, while the oral mucosa is generally considered more permeable than skin, the buccal mucosa generally acts as a barrier limiting the permeation of released active ingredient (eg, large molecules). ${ }^{10}$ Hydrophilic compounds and large or highly polar molecules follow paracellular transport, whereas lipophilic drugs typically follow transcellular transport through the lipid bilayer. ${ }^{6}$ Consequently, increased retention of the mucoadhesive drug formulation on the buccal mucosa at the site of action, ie, oral cavity (by virtue of the delivery vehicle design), would overcome the mucous barrier layer and enhance drug partition to the local target tissue. Such approach constitutes the primary strategy for effective sustained localized activity of targeted therapeutic compounds. ${ }^{8,9}$

Mucoadhesive buccal tablets would be a consummate delivery platform for local and sustained delivery of chemopreventive agents and antineoplastic therapeutics, to target proliferating oropharyngeal cancers. Our candidate drug molecule, genistein (4',5,7-trihydroxyisoflavone) (Gen), is a small biologically active flavonoid identified as the predominant isoflavone in soy products. ${ }^{11}$ Multiple pharmacological anticancer activities have been identified for Gen, mainly: protein tyrosine kinase inhibition, topoisomerase II inhibition, antioxidant activities, G2/M phase cell cycle inhibition, induced differentiation, and deregulation of mitochondrial membrane pore permeability. ${ }^{11-14}$ Furthermore, Gen has been demonstrated to modify the activity of key cell proliferation and survival pathways, such as those controlled by protein kinase B, signal transducers and activators of transcription (STAT) protein, nuclear factor-kB, and cyclooxygenase-2. ${ }^{15,16}$ Most importantly, Gen has been recently reported to trigger apoptosis in different solid and hematological cancer models, ${ }^{11}$ mediated by activation of caspase-9 and -3. Proapoptotic effects of Gen were also associated with mitochondrial depolarization and cytosolic release of cytochrome $\mathrm{c}$, in T-cell lymphoma and acute promyelocytic leukemia cells as well as in human prostate, breast, and hepatic carcinoma cultures. ${ }^{14,17-19}$

Despite evident anticancer activity of Gen, its use is limited by its lipophilic nature, extremely low aqueous (AQ) solubility, ${ }^{20}$ and extensive metabolism, resulting in poor bioavailability and pharmacokinetic profiles. Thus, nanoscale vehicles have been recently designed and investigated to enhance the solubilization and delivery of Gen for various therapeutic applications, such as antioxidant and cancer chemoprevention. ${ }^{21}$ For instance, nanoemulsified carriers of Gen showed significant improvements in both oral and transdermal bioavailabilities, ${ }^{21,22}$ owing to superior drug encapsulation efficiencies, as demonstrated through Genloaded micelles ${ }^{23}$ and Gen hydrogel-based micro/nanoscale emulsions, respectively. ${ }^{22,24}$ Very recently, we successfully developed various optimized Gen-loaded nanocarriers as targeted cancer therapy. Liposomal vehicles of Gen markedly induced apoptosis in different solid tumor cell lines of diverse origin, leading to both time- and concentration-dependent improvement in cancer cell repression. ${ }^{18}$ Further enhancement of the antiproliferative efficacy of Gen was achieved using surfactant-based vesicles (specifically, polymeric lipid micelles and nanoemulsions [NEs]). ${ }^{19}$ In the latter nanocarrier system designs, the active Gen drug molecules served 
as therapeutic cargo as well as in a mitochondria-homing moiety, intrinsically inducing extensive apoptosis in two different human cancer models in vitro. ${ }^{19}$

Our current project employs NEs that rely on active amphiphilic tocopheryl moieties, which were utilized previously, ${ }^{25}$ successfully loaded with the proapoptotic lipophilic drug candidate Gen. Additional mucoadhesion properties - acquired via chitosan (Chito) layering of Gencontaining NE particles - were investigated to produce pharmaceutically stable and acceptable mucoadhesive buccal platforms, namely liquid sprays and solid tablets. One of the major advantages of using multilayer NEs as delivery systems is that the properties of the Chito interfacial layer surrounding the oil/emulsifier interior can be tuned through careful design of system composition and preparation conditions. ${ }^{26}$ This in turn permits further control of the overall performance of such drug formulation. ${ }^{27}$ The aim of our mucoadhesive lipophilic nanosystem was to extend both the retention and the release profile of the therapeutic drug molecule Gen, within the oral cavity. Our Chito-coated nanoemulsified system (in liquid or tablet dosage form) would, not only enhance the anticancer drug partitioning to oral mucosal membrane but also, mediate the targeted delivery of Gen at the site of action, ie, directly onto oral, pharyngeal, and tongue cancerous lesions. Our proof-of-concept mucoadhesive pharmaceutical nanocomposites represent potential development of adjuvant maintenance/preventive therapy for patients who have already undergone cancerous lesion resection in the oral cavity and oropharyngeal region.

\section{Material and methods Materials}

Cold-pressed, high omega-3 fatty acid-containing chia seed oil (United States Department of Agriculture [USDA]-certified) was obtained from Foods Alive (Waterloo, IN, USA). Tween $^{\circledR} 80$ (T80), trypsin/ethylenediaminetetraacetic acid (EDTA), penicillin/streptomycin, and fetal bovine serum were obtained from Thermo Fisher Scientific Inc. (Waltham, MA, USA). Powdered Chito (medium molecular weight [MW], dry matter content $\geq 90 \%$, ash content $<0.5 \%$, and degree of acetylation $85 \%$, MW range 100-300 KD), Avicel ${ }^{\circledR}$ $\mathrm{PH}-101$ (cellulose microcrystalline [MCC]) powder), and trypan blue assay reagent were purchased from Sigma-Aldrich Corp (St Louis, MO, USA). Tween 20, CellTiter Blue ${ }^{\circledR}$ (Promega, Madison, WI, USA), DL- $\alpha$-tocopherol (also known as vitamin E, VE), and BD-Black 96-well tissue culture plates. While Gen was purchased from LC Laboratories (Woburn, MA, USA), national formulary (NF)-grade tocopheryl polyethylene glycol succinate ester (TPGS) (Speziol ${ }^{\circledR}$ TPGSPharma) and Solutol ${ }^{\circledR}$ HS-15 (SHS15) were generously supplied by Cognis (Ludwigshafen, Germany) and Muchler Inc./BASF (Cincinnati, OH, USA), respectively. Human tongue squamous cell carcinoma (SCC-4 cell line) cells and pharyngeal squamous cell carcinoma (FaDu cell line) cells were purchased from American Type Culture Collections (Manassas, VA, USA).

\section{Preparation of mucoadhesive NEs}

Vehicle NEs were prepared using polyunsaturated fatty acid (PUFA)-rich chia seed oil and $\alpha$-tocopherol as the matrix for the oily component of NE. ${ }^{25}$ NEs were prepared by coarse homogenization, followed by 30 minutes of lowamplitude ultrasonication (under cooling and $\mathrm{N}_{2}$ gas). ${ }^{28}$ Briefly, to the oily phase (composed of 30:70 wt $\%$ of chia seed oil:VE), the surfactant blend (SHS15:VE-TPGS as 40:60 wt $\%$ ) was added, while warming and gently mixing on a vortex mixer, at $800 \mathrm{rpm}$, at $50^{\circ} \mathrm{C}$ for 2 minutes. For drug-loaded formulations, Gen $(2 \mathrm{mg} / \mathrm{mL}$ dissolved in $200-$ proof ethanol) was mixed into the oil/cosolvent mixture, followed by subsequent rotary evaporation of the solvent. ${ }^{19}$ The produced homogeneous oil-surfactant mixture was hydrated with $0.4 \%$ Chito in $0.5 \%$ acetic acid AQ solution (adjusted $\mathrm{pH} \sim 5.7$ ), followed by brief vortex mixing and then, homogenization for 5 minutes at 20,000 rpm, using a Ultra Turrax-10 homogenizer (IKA Works Inc., NC, USA) to produce the layered, coarse oil-in-water emulsion. Subsequently, microemulsions were ultrasonicated at 6 watts power, using a Misonix XL2000 P-6 low amplitude microprobe sonicator (Qsonica; Newtown, CT, USA) for three cycles (each sonication cycle time was divided as 10 minutes on plus 3 minutes off), to obtain the nanosized oil droplets (average $\eta=17 \pm 2.4 \mathrm{cP}$, with final concentration of Chito in NEs $=0.3 \mathrm{wt} \%$ ). Finally, resultant Chito-layered NEs were washed with a MicroKros ${ }^{\circledR}$ tangential flow filtration manual system (Spectrum Laboratories Inc., Rancho Dominguez, CA, USA) containing modified polyethersulfone hollow fiber modules (MW cutoff $[\mathrm{MWCO}]=300 \mathrm{KD}$ ), while maintaining the total NE sample volume by deionized $\mathrm{H}_{2} \mathrm{O} .{ }^{29}$ Control primary (AQ) NEs were produced in the same manner, except that deionized distilled water was used instead of Chito solution (ave $\eta=1.8 \pm 0.6 \mathrm{cP}$ ) in the first hydration step. ${ }^{19}$

\section{Tablet preparation}

Tablets were prepared from homogeneous paste (composed of freeze-dried NEs, MCC, and dextrose, in 1:3:0.5 ratio) via 
direct compression method. The prepared blend (200 mg) of each formulation (control vs Chito-coated vehicles, as drugfree and Gen-loaded NEs) was compressed, using a TPD-0 manual 1-station single-punch tablet press machine (Yangzhou Nuoya Machinery Co. Ltd, Jiangsu, People's Republic of China) at a maximum pressure of 1.5 ton, forming a singlelayered, flat-faced tablet of $7 \mathrm{~mm}$ diameter. The tablets were left to air-dry overnight before further testing. ${ }^{30}$

\section{Physicochemical characterization of Chito-coupled NEs}

All formulations were characterized for mean particle size, size distribution, and zeta $(\zeta)$-potential, using the dynamic light scattering technique, with a Malvern Zetasizer analyzer (NanoZS ${ }^{\circledR}$; Malvern Instruments Inc., Malvern, UK), at $273^{\circ}$ fixed angle and at $25^{\circ} \mathrm{C}$ temperature.

\section{For particle size analysis}

All NEs samples were diluted with deionized distilled water before analysis, and the volume average oil droplet hydrodynamic diameter and the polydispersity index (PDI) were determined. ${ }^{19,25}$

\section{For the $\zeta$-potential measurements}

NE samples were diluted with deionized distilled water and placed in the electrophoretic cell of the Malvern nanosizer, then the average surface charge was determined. ${ }^{19,25}$

\section{$\mathrm{pH}$-dependent stability}

NE formulations were $50 \times$ diluted with deionized distilled water, and the mean droplet size and $\zeta$-potential were determined after 30 minutes, following $\mathrm{pH}$ adjustment (ranging from 3-9) of the final dilution (data not shown). ${ }^{27}$

\section{Turbidimetric method}

This NE-modified procedure was recognized as a tool to predict and evaluate micro/nanoemulsion stability based on controlled rapid dilution, which is typically unable to break the emulsified systems. ${ }^{25}$ For turbidity, $0.05 \mathrm{~mL}$ of each sample, stored for 60 days at $4^{\circ} \mathrm{C}$, was diluted to $25 \mathrm{~mL}$ with deionized distilled water, and the percentage transmission (\%T) was measured at $600 \mathrm{~nm}$, using a Perkin Elmer Lambda EX210 UV/visible spectrophotometer (San Jose, CA, USA). With the blank deionized distilled water control set at $100 \% \mathrm{~T}$, the turbidity of the diluted NE was calculated as below $(n=4):^{25}$

$$
\text { Turbidity } \%=100-\% \mathrm{~T} \text {. }
$$

\section{Analysis of Gen-incorporation in nanovesicles}

High-performance liquid chromatography (HPLC) analytical method was used to determine the levels of Gen incorporated in the nanolipidic formulations. Formulation samples $(20 \mu \mathrm{L})$ were injected through an ACE PFP C-18 column $(4.6 \times 250 \mathrm{~mm}$, $4 \mu \mathrm{m}$ packing volume), using degassed methanol:0.1\% acetic acid in $\mathrm{H}_{2} \mathrm{O}(55: 45 \mathrm{vol} / \mathrm{vol})$ as the mobile phase. The flow rate of the mobile phase was maintained at $0.5 \mathrm{~mL} / \mathrm{minute}$, and ultraviolet (UV)-detection was performed at $261 \mathrm{~nm}$. The Gen concentration in the sample was determined using a calibration curve of Gen dissolved in methanol. The HPLC method showed excellent reproducibility, with precision of less than $3 \%$ relative standard deviation (RSD) as well as excellent accuracy, between $92.3 \%$ and $99.94 \%$, for Gen. The lower limit of Gen quantitation was $0.08 \mu \mathrm{g} / \mathrm{mL}$. The drug encapsulation efficiency in the NE formulations was determined by ultrafiltration technique ( $\mathrm{MWCO}=3,000 \mathrm{Da})$, using centrifugal filter devices (Centricorn, Millipore, Bedford, MA, USA), followed by HPLC quantification. ${ }^{18,19,21}$

\section{Stability of NEs and incorporated Gen}

Physical stability of nanoformulations was assessed over a period up to 3 months $\left(\mathrm{D}_{90}\right)$ upon storage at low temperature $\left(4^{\circ} \mathrm{C} \pm 1^{\circ} \mathrm{C}\right)$. During this storage period, the particle size and size distribution were evaluated, as was the as creaming index (CI) of the NEs (data not shown). ${ }^{25}$ The chemical stability of incorporated drugs was determined, via HPLC, as the entrapment efficiency/drug recovery (\%) (after storage), which was determined using the following formula: ${ }^{21}$

$\begin{aligned} & \text { Drug } \\ & \text { recovery }(\%)\end{aligned}=\frac{\text { Amount of Gen in nanoformulation, } \mathrm{D}_{60}}{\text { Amount of Gen in nanoformulation, } \mathrm{D}_{0}} \times 100$,

where $\mathrm{D}_{0}$ and $\mathrm{D}_{60}$ refer to initial and post-60 days measurement, respectively. These parameters were employed as a tool to evaluate the overall nanosystem stability. ${ }^{19,25}$

\section{Buccal tablets physical analysis}

Hardness test

The crushing strength $\left(\mathrm{N} / \mathrm{cm}^{2}\right)$ of tablets was determined using a modified Extech digital compression force gauge/ tester (Extech Instruments Corp, Nashua, NH, USA). ${ }^{31}$

\section{Friability test}

This was determined for 10 tablets/formulation, after rotating them in a friabilator's plastic cylinder (Gowe ${ }^{\circledR}$ Industrial, 
Suzhou, People's Republic of China), vertically at $25 \mathrm{rpm}$ for 4 minutes. After dusting, the total remaining weight of the tablets was recorded, and the percent friability was calculated (\% loss in weight). ${ }^{32}$

\section{In vitro swelling studies}

Buccal tablets of each formulation $(n=6)$ were individually weighed (W1), then were placed separately in Petri dishes containing $2 \%$ agar gel, kept in an incubator at $37^{\circ} \mathrm{C} \pm 0.5^{\circ} \mathrm{C}$, and examined for any physical change. At regular intervals $(0.5,1,2$, and 4 hours $)$, the tablets were removed from Petri dishes and excess water removed carefully using filter paper. The swollen tablets were then reweighed (W2); the swelling index (SI) of each tablet formulation was calculated using this formula: ${ }^{32}$

$$
\mathrm{SI}=(\mathrm{W} 2-\mathrm{W} 1) / \mathrm{W} 1 \text {. }
$$

\section{Surface $\mathrm{pH}$ measurements}

In order to estimate the potential for local irritation to the buccal mucosa upon extended adhesion at site of action, surface $\mathrm{pH}$ was determined. From each formulation, six tablets were allowed to swell for 8 hours on the surface of a $2 \%$ agar plate, as described. The surface $\mathrm{pH}$ was measured by using a $\mathrm{pH}$ paper placed on the surface of the swollen tablet. ${ }^{31}$

\section{In vitro release studies}

Liquid NE buccal spray platform

Gen-loaded NE samples $(0.3 \mathrm{~mL})$ were introduced into a dialysis tube (MWCO =7,000-10,000 Da) (Spectra/Por-Spectrum Laboratories Inc., Rancho Dominguez, CA, USA), which was then suspended into $1 \mathrm{~L}$ of release medium (phosphate-buffered saline [PBS] pH 7.4) containing $0.05 \%$ Tween $20 .^{18,19}$ The system was stirred at $300 \mathrm{rpm}$ at $37^{\circ} \mathrm{C} \pm 0.5^{\circ} \mathrm{C}$. As a negative control, a dialysis bag containing free solution of Gen (Gen-Sol) (at equivalent amount $\approx 2.2 \mathrm{mg}$, dissolved in $2 \mathrm{~mL}$ ethanol: PBS at 90:10 v/v) was included to confirm that complete dissolution of drug/minimal sink condition was achieved. At predetermined time intervals, $1 \mathrm{~mL}$ samples were withdrawn and replaced with an equal volume of fresh release medium. The amount of Gen released was measured by HPLC as described above. Data were reported as mean with standard deviation (SD) $(n=3) \cdot{ }^{21,23}$

\section{Solid buccal tablet platform}

NE stability release in AQ media was determined via the HPLC method mentioned above. Briefly, drug-containing NE tablet samples were placed in a floating bucket of a Unites
States Pharmacopeia (USP) type-2 dissolution apparatus (VK 7000; Varian, Cary, NC, USA), in 50-100 mL simulated saliva solution that consisted of PBS ( $\mathrm{pH}$ 6.75), with $300 \mathrm{rpm}$ paddle stir speed. Similar to the liquid NE protocol, containing Gen-Sol (at equivalent amount $\approx 2.2 \mathrm{mg}$ ) was also included as a negative control, to confirm that complete dissolution of drug/minimal sink condition was achieved. Every 5 minutes, aliquots of AQ solution were drawn and analyzed, via the abovementioned HPLC assay, for the quantity of Gen released in the external medium. ${ }^{30}$

\section{Ex vivo residence time}

Using a modified USP 2 dissolution apparatus (Varian VK 7000), the PBS ( $\mathrm{pH} 5.75$ ) was used as dissolution medium, maintained at $37^{\circ} \mathrm{C} \pm 0.5^{\circ} \mathrm{C} .{ }^{33} \mathrm{~A}$ segment of porcine buccal mucosa, of $4 \mathrm{~cm}$ length, was glued to the surface of a glass slide, which was then vertically attached to the apparatus. Three tablets of each formulation were hydrated using $15 \mu \mathrm{L}$ of PBS buffer on one side, and the hydrated surface was brought into contact with mucosal membrane. Each tablettissue complex was secured on a glass slide and completely immersed in the buffer solution, with the paddle adjusted at a distance of $5 \mathrm{~cm}$ from the tablet, at $50 \mathrm{rpm}$ rotation speed. The time for complete erosion or detachment from the mucosa was recorded. ${ }^{33}$

\section{Measurement of mucoadhesive strength}

Freshly excised porcine buccal mucosa, obtained from a slaughterhouse, was utilized as the model substrate, along with PBS (pH 6.6), and employed as moistening fluid. A compressed buccal tablet was horizontally placed or "sandwiched" between two layers of excised model tissue substrates, with constant weight of $50 \mathrm{gm}$ on top, for a total constant period of 10 minutes. Bioadhesive strength was measured in terms of the force required to detach the tablet from the animal buccal mucosa, using an Extech 475040 Force Gauge Meter (Extech Instruments Corp). The upward tension was arrested when the tablet was detached from the porcine buccal mucosa, and the corresponding force reading was registered. ${ }^{30,33}$

\section{In vitro cell culture}

SCC-4 cells, FaDu cells, and murine connective tissue fibroblasts (L929) (American Type Culture Collection) were grown in Roswell Park Memorial Institute (RPMI) 1640, Dulbecco's Modified Eagle's Medium (DMEM)/High, and Eagle's minimal essential medium (EMEM) culture media, respectively, containing $10 \%$ fetal bovine serum 
and penicillin $(100 \mathrm{U} / \mathrm{mL}) /$ streptomycin $(100 \mu \mathrm{g} / \mathrm{mL})$, in a humidified environment of $37^{\circ} \mathrm{C}, 5 \% \mathrm{CO}_{2}$. Cells were seeded at a concentration of $6 \times 10^{3}-1.5 \times 10^{4}$ cells $/ \mathrm{cm}^{2}$ and subcultured at approximately $70 \%-80 \%$ confluency. Cells between passages 8 to 20 were used for experimentation. ${ }^{25,28}$

\section{Trypan-blue exclusion biocompatibility assay}

Normal L929 cells were seeded in 6-well plates, at density of $2 \times 10^{5}$ cells/well, until $70 \%$ confluent. Cells were then incubated with control media, drug-free primary (AQ), and Chito-coated chia seed oil NEs (prepared with surfactant mixtures of TPGS, mixed with either SHS15 or T80 (in weight ratio of $2: 3$ ) in serum-free media, at concentration of $0.75 \mathrm{mg} / \mathrm{mL}$ of the oily phase. After 24 and 48 hours respectively, media containing the NEs were removed, and cells were washed with Hank's balanced buffer (HBSS) and incubated in corresponding complete media overnight before performing the trypan blue-exclusion viability assay. Finally, trypsin-detached cells were treated with $0.4 \%$ trypan blue $(50 \mu \mathrm{L})$ dye after enzyme neutralization. Live cells were counted using a hemocytometer ( $\mathrm{n}=3$ aliquots) under light microscope, and averages of three runs were calculated. ${ }^{25}$

\section{Cytotoxicity assays}

Human cancer cell lines SCC-4 and FaDu were seeded at $10 \times 10^{3}$ cells per well of 96 -well plates, in four replicates, for 24 hours. Then, the respective complete culture media were exchanged for serum-free media containing several twofold serial dilutions of selected Gen-free/plain and Gen-containing NE formulations - either as AQ or Chito-layered NEs. At certain coincubation time points (12, 24, and 48 hours), after washing twice with HBSS, cell viability was determined using a CellTiter Blue ${ }^{\circledR}$ Kit and reading sample plate fluorescence at excitation $\lambda=480 \mathrm{~nm}$ and emission $\lambda=530 \mathrm{~nm}$, using a Synergy 2 BioTek fluorescence plate reader (BioTek Instruments Inc, Winooski, VT, USA), according to manufacturer's instructions. ${ }^{18,25}$

\section{Data analysis}

A minimum of triplicates were run for each experiment. Data were reported as mean $\pm \mathrm{SD}$. Comparisons between the groups were made using Student's $t$-test, and for more than two groups, nonparametric analysis of variance (ANOVA) (Kruskal-Wallis test, with Tukey's post hoc analysis) was used to compare results. The $P<0.05$ values were considered statistically significant. All statistical analyses were performed using GraphPad Prism software (version 5.0; GraphPad Software, Inc., La Jolla, CA, USA). ${ }^{18,19}$

\section{Results and discussion}

Owing to the lipophilic nature of Gen, it was likely to become an ideal candidate for loading into lipid-based nanocarriers, namely NEs, as noted earlier. ${ }^{18,19}$ Combined with mucoadhesive coating, the optimized nanoemulsification of Gen would allow for sustained delivery of this antiproliferative drug candidate into oral carcinoma lesions, due to improved solubilization and permeation through biomembranes. ${ }^{26,34}$

\section{Development of mucoadhesive NE formulations of Gen}

Several reports of successful nanoemulsification of active isoflavones, such as Gen and quercetin, were in tandem with our earlier NE formulations of Gen using PUFA-rich vegetable oils. ${ }^{19,24}$ In our current NE design, chia seed oil was employed as the core oil matrix for incorporation of Gen. Chia (Salvia hispanica) is a biannual plant native to South America, mostly cultivated for its seeds, which contain high levels of $\omega-3$ and $\omega-6$ PUFAs, as well as fibers and proteins. ${ }^{35,36}$ Notably, the fatty acid component of this oil tends to be mostly $\omega-3$ fatty acids (approximately 60\% overall, mostly as $\alpha$-linolenic acid) and some $\omega-6$ fatty acids (20\% in general, mostly as $\gamma$-linolenic acid). ${ }^{37}$ In addition, some active phenolic compounds are present in chia seed oil, with "myricetin", an antioxidant flavonol, being the most plentiful one. ${ }^{36,37}$ The oil phase of the NE was composed of a homogeneous mixture (70:30 wt $\%$ ) of chia seed oil mixed with $\alpha$-tocopherol, respectively. Within the oily matrix, $\alpha$-tocopherol was included as an integral part of our NE system, providing important advantages: $\alpha$-tocopherol provides antioxidant preservation of PUFAs in chia oil during ultrasonication and/or high-shear extrusion steps; plus, it facilitates emulsification of the entire NE oil core, through mutual interaction with the tocopherylportion of TPGS, a component of our test surfactant blend. ${ }^{19,25}$ As seen in Table 1, different nonionic surfactants were used to produce the NE (average size 85-140 nm), using SHS15, T80, and TPGS, either alone or mixed together. An average calculated hydrophilic-lipophilic balance (HLB) value $\geq 13.5$ for the emulsifier blend was found to be quite sufficient to successfully develop several prototype NEs, with a narrow PDI $\left(<0.4\right.$ [data not shown]). ${ }^{19,25}$

Figure 1 illustrates the stepwise buccal tablet formulation scheme, where Gen was first incorporated into the homogeneous oil phase-emulsifier mixture using 100\% ethanol as the common cosolvent, followed by solvent evaporation. 
Table I Physicochemical characterization and stability of Gen-loaded NE formulations, representing chitosan layering and different surfactant blend compositions

\begin{tabular}{llllll}
\hline NE (surfactant mix, wt\%) & Chitosan & Genistein content $(\mathbf{m M})$ & Size $(\mathbf{n m})$ & $\zeta$-potential $(\mathbf{m V})$ & Drug retention $\left(\mathbf{D}_{60}, \%\right)$ \\
\hline SHSI5 (I00) & + & $19.8 \pm 3.4$ & $94.1 \pm 4.3$ & $+14.3 \pm 4.8$ & $83.2 \pm 4.3$ \\
SHSI5:TPGS (60:40) & - & $20.2 \pm 3.7$ & $87.9 \pm 7.5$ & $-26.4 \pm 5.2$ & $84.5 \pm 4.6$ \\
& + & $22.6 \pm 7.2$ & $118.2 \pm 14.7$ & $+18.9 \pm 3.3$ & $93.6 \pm 2.0$ \\
SHSI5:TPGS (40:60) & - & $22.4 \pm 4.3$ & $113.9 \pm 12.3$ & $-28.1 \pm 4.1$ & $94.2 \pm 0.8$ \\
& + & $24.6 \pm 5.4$ & $128.4 \pm 13.1$ & $+20.3 \pm 2.6$ & $94.6 \pm 2.1$ \\
T80 (I00) & - & $25.2 \pm 6.6$ & $122.8 \pm 9.3$ & $-25.6 \pm 3.1$ & $95.2 \pm 1.6$ \\
& + & $24.8 \pm 5.1$ & $87.9 \pm 9.8$ & $+19.3 \pm 4.1$ & $86.4 \pm 2.7$ \\
T80:TPGS (60:40) & - & $25.3 \pm 4.8$ & $92.5 \pm 11.3$ & $-26.4 \pm 2.8$ & $89.2 \pm 3.6$ \\
& + & $26.5 \pm 3.9$ & $118.6 \pm 8.4$ & $+23.3 \pm 3.5$ & $94.9 \pm 0.9$ \\
T80:TPGS (40:60) & - & $27.3 \pm 5.7$ & $110.3 \pm 11.3$ & $-28.4 \pm 3.6$ & $95.4 \pm 2.2$ \\
& + & $24.1 \pm 4.2$ & $138.7 \pm 18.3$ & $+24.3 \pm 2.8$ & $96.8 \pm 0.8$ \\
TPGS (I00) & - & $24.4 \pm 6.7$ & $129.5 \pm 17.3$ & $-27.5 \pm 3.9$ & $96.3 \pm 1.3$ \\
& + & $23.2 \pm 8.5$ & $136.3 \pm 17.4$ & $+21.3 \pm 4.8$ & $88.7 \pm 2.4$ \\
\hline
\end{tabular}

Notes: Values are expressed as mean $( \pm S D) n=5-6$.

Abbreviations: $\mathrm{D}_{60}$, post-60 days measurement; Gen, genistein; NE, nanoemulsion; SD, standard deviation; SHSI5, Solutol ${ }^{\circledR}$ HS; T80, Tween ${ }^{\circledR} 80$; TPGS, tocopheryl polyethylene glycol succinate.

Cationic NE droplets $(\sim 120 \mathrm{~nm}$; $\zeta$-potential $\sim+18 \mathrm{mV}$ to $+26 \mathrm{mV}$ ) were obtained after ultrasonication of intermediate macroemulsions, which were produced following the addition of the Chito-containing AQ/external phase (final concentration $0.3 \mathrm{wt} \%$ ). Afterwards, lyophilized Chitolayered nanodroplets were mixed with inert tablet excipients (eg, MCC and dextrose), which were finally pressed into a Gen tablet dosage form intended for buccal applications.

The inclusion of $0.3 \mathrm{wt} \%$ Chito in the AQ phase successfully switched the $\zeta$-potential profile of all test formulations to be cationic, regardless of the amplitude of the native negative charge (Table 1). While this strongly indicates successful and complete coating of the nanoemulsified droplets (masking original negative charges of all surfactants in mixed-micelle coronas), the almost stable value of the cationic surface $\zeta$-potential of the Chito-coated droplets $(+22.6 \pm+3.4 \mathrm{mV})$ suggests a near saturation-level stability of their Chito polymer overcoat. ${ }^{27,38}$ This effect was further delineated through data presented in Figure 2, illustrating the effect of Chito polyelectrolyte density on both the mean NE droplet size and surface charge. An apparent concentrationdependent rise in the average droplet $\zeta$-potential, until nearly a plateau was reached in all NEs at Chito polymer concentrations $\geq 0.3 \mathrm{wt} \%$, corroborate this conclusion (Figure 2B). Consequently, such steady cationic surface charge seemed to result in smaller and more stabilized NE particles, leading to well-dispersed small NE droplets (approximate diameter 100-140 nm), as layered Chito concentrations exceeded $0.25-0.3 \mathrm{wt} \%$, similar to earlier reported findings (Figure $2 \mathrm{~A}$ ). ${ }^{27,39}$
When comparing the different prototype NE formulations (Table 1) in regards to the composition of the surfactant blend used, several key points can be observed. Irrespective of the polyethoxylated cosurfactant (T80 or SHS15), the inclusion of the chromane multiring structure of TPGS within the mixed-micelle emulsifiers (notably at the higher weight ratio of $60 \%$ ) seemed to induce a rather larger droplet diameter. Hence, TPGS mixed emulsifiers can mediate higher Gen-loading capacity and improved drug retention in comparison with single-component emulsifiers, without producing a substantial change of NE $\zeta$-potential, at the final fixed $\mathrm{pH}$ of 5.7. In complete agreement with previous data, ${ }^{27,40}$ for Chito-layered micro/nanoscale emulsions, the particle electrical charges were found to be inversely dependent on the final adjusted $\mathrm{pH}$ value. As $\mathrm{pH}$ increased, the cationic charge decreased until neutral or slightly anionic ( $\approx-7.5 \mathrm{mV}$, at $\mathrm{pH}>7.0$ ). On the other hand, alteration of the final $\mathrm{pH}$ had almost no effect on droplet diameter, in all $\mathrm{NE}$ samples, until $\mathrm{pH}$ values exceeded 7.0, when particle sizes effectively inflated (five- to sevenfold), indicating instability and increased aggregation owing to diminished surface charges (data not shown). ${ }^{26}$

As seen from Figure 3, both the turbidity and CI values, measured after 60 days, correlated very well with the previously noted behavior of PUFA-rich argan oil-based NEs. ${ }^{25,28}$ Here, primary chia oil NEs containing a monocomponent surfactant system, are present at either end of the calculated final HLB range of all developed NEs (13.5> HLB $\geq 15.0$ ). Improved physical stability of nano/microemulsions has been repeatedly achieved through Chito polyelectrolyte 


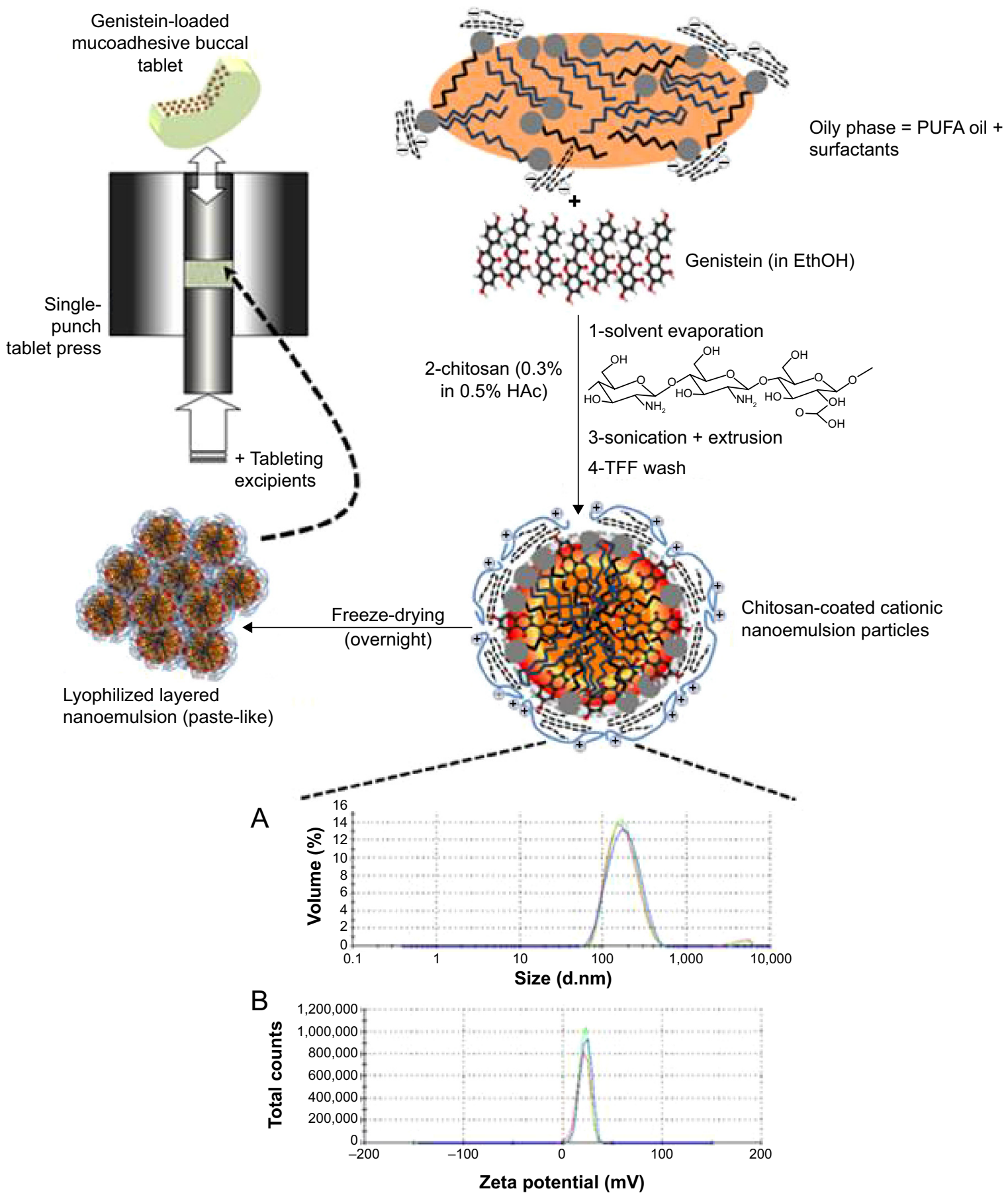

Figure I Formulation process of genistein-loaded mucoadhesive buccal tablets.

Notes: Representative scheme of preparation of chitosan-coated NEs (incorporating Gen as the active ingredient), which is pressed with excipients into solid tablets, following flow filtration and lyophilization. Included graphs demonstrate $(\mathbf{A})$ average particle diameter $(\mathrm{nm})$ and $(\mathbf{B})$ surface charge $(\zeta$-potential in $\mathrm{mV})$, obtained for the prototype layered NE.

Abbreviations: Gen, genistein; HAc, acetic acid; NE, nanoemulsion; PUFA, polyunsaturated fatty acid; TFF, tangential flow filtration.

layering, possibly due to the typical higher final viscosity compared with primary/AQ NEs ( $\eta$ was estimated to be eight- to ninefold higher in the current study). ${ }^{27,41}$ Despite some improvement in stability of mixed-emulsifier with $0.3 \%$ layered Chito NEs (Figure 3A), the CI of single emulsifier-based samples remained substantially elevated, coupled with a reduction in $\% \mathrm{~T}$, upon acute dilution. Such high CI and turbidity (\%) values - the selected indicators for physical stability of pharmaceutical NE systems - suggest inferior cold shelf storage stability, $\leq 2$ months, in the case of coated NEs that are composed of a single nonanionic emulsifier (T80, TPGS, or SHS15). ${ }^{25}$ To this effect, after 
A

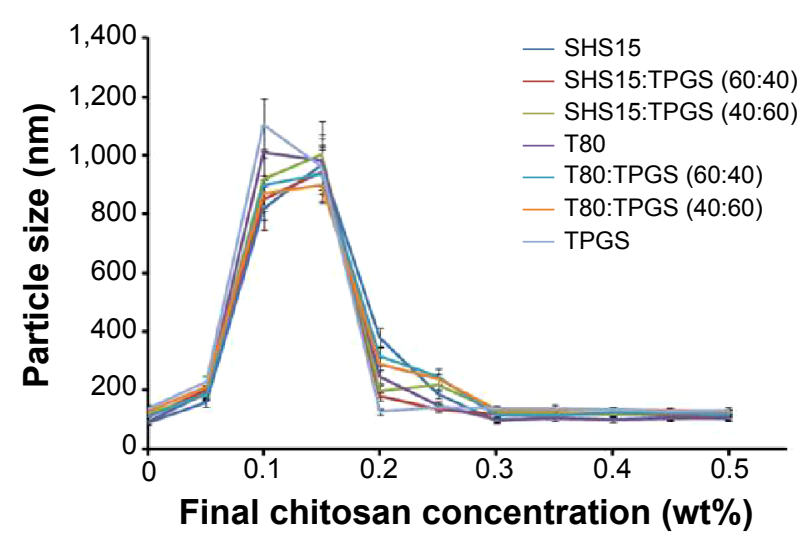

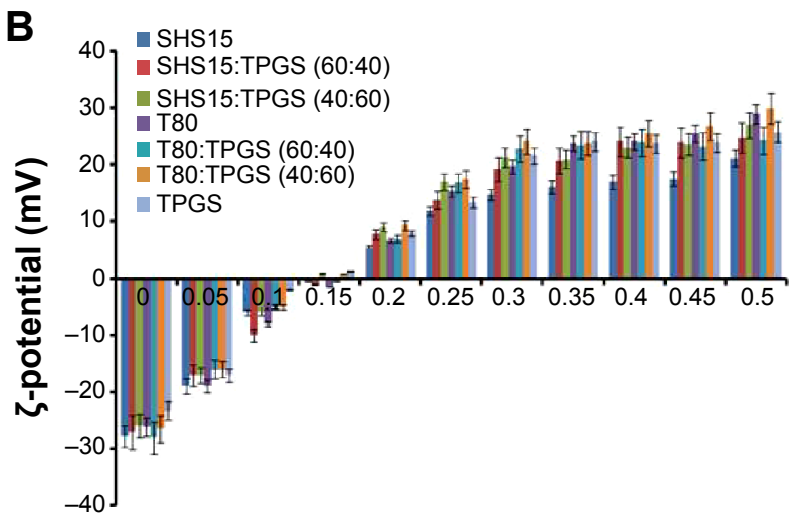

Final chitosan concentration (wt\%)

Figure 2 Change in (A) mean droplet size and (B) electrical charge (measured as $\zeta$-potential), of the various NE formulations, with the increasing concentration of cationic chitosan solution (0-0.5 wt\%) as the external layer, at $\mathrm{pH}$ 5.7.

Notes: Values are shown \pm SD. $n=6$.

Abbreviations: NE, nanoemulsion; SD, standard deviation; SHSI5, Solutol ${ }^{\circledR}$ HS; T80, Tween ${ }^{\circledR} 80$; TPGS, tocopheryl polyethylene glycol succinate.

A

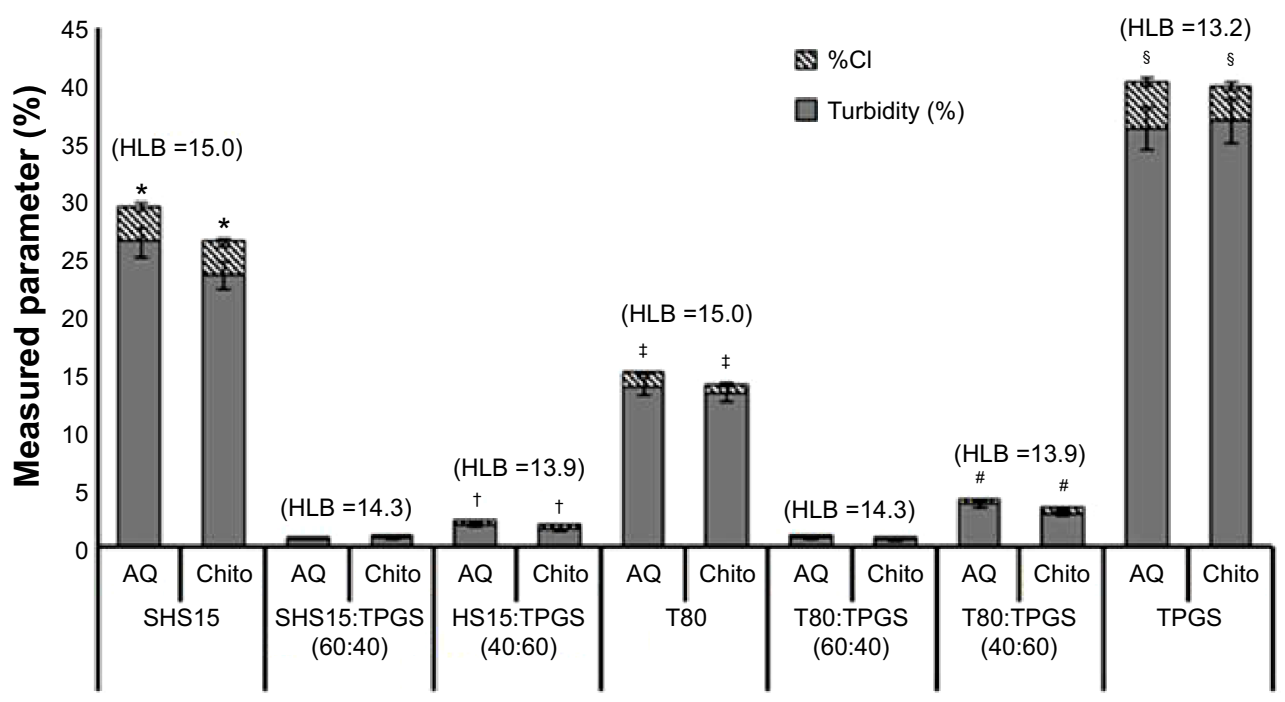

B

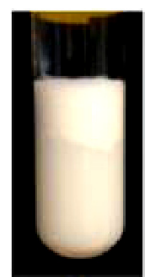

(1)

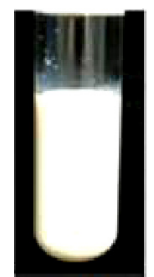

(2)

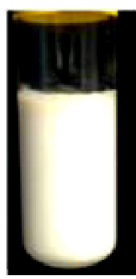

(3)

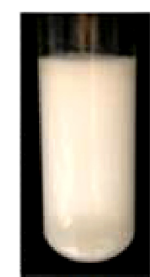

(4)

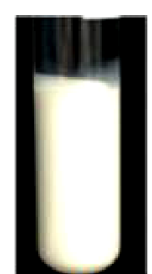

(5)

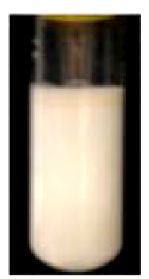

(6)

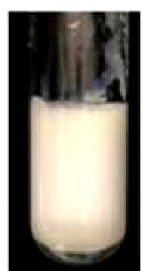

(7)

Figure 3 Physical stability of the different test NE formulations.

Notes: (A) The relation between formulation stability (indicated via sudden dilution turbidimetry \%, and $\mathrm{Cl}$ and overall $\mathrm{HLB}$ value, calculated for the surfactant blends $(0$, $40: 60,60: 40$, or 100 wt\%) present in various prepared NE formulations, of both types - primary/AQ or layered/Chito NE. Values are shown with \pm SD. Mean values with unlike superscripts $(*, \#, \S, \dagger, \ddagger)$ are statistically different $(P \leq 0.05) n=4-6)$. (B) Photographs of chitosan-coated chia seed oil-based NEs after 72 hours of shelf-storage at room temperature: (I) NE with SHSI5 as emulsifier at 100 wt\%; (2) NE with SHSI5/TPGS emulsifier at 60/40 wt\%; (3) NE with SHS I5/TPGS emulsifier at 40/60 wt\%; (4) NE with T80 as emulsifier at 100 wt\%; (5) NE with T80/TPGS emulsifier at 60/40 wt\%; (6) NE with T80/TPGS emulsifier at 40/60 wt\%; and (7) NE with TPGS as emulsifier at $100 \mathrm{wt} \%$.

Abbreviations: AQ, aqueous; Chito, chitosan; Cl, creaming index; HLB, hydrophilic-lipophilic balance; NE, nanoemulsion; SD, standard deviation; SHSI5, Solutol ${ }^{\circledR} \mathrm{HS}-\mathrm{I5}$; T80, Tween ${ }^{\circledR} 80$; TPGS, tocopheryl polyethylene glycol succinate. 
72 hours of shelf storage at $23^{\circ} \mathrm{C}$, considerable creaming was visible in both AQ NEs and Chito-coated NEs made with 100 wt $\%$ SHS15 as emulsifier (Figure 3B). Likewise, apparent phase separation was noted in both primary/AQ and Chito-coated NEs prepared using T80 or TPGS as sole emulsifiers (Figure 3B) but was drastically less visible in the corresponding NEs containing the 40/60 wt $\%$ of $\mathrm{T} 80 /$ TPGS emulsifier blend (Figure 3B).

Prototype formulations with the lowest physical instability marker values (containing surfactant blend, composed of $40 \%$ of TPGS, mixed with $60 \%$ of either SHS15 or T80) were selected for longer-term storage stability characterization, which extended up to 90 days (Figure 4A), as well as for lyophilization-reconstitution study (Figure 4B).

Polyelectrolyte layering of emulsions was proposed to enhance both the physical and chemical stability of encapsulated unsaturated oil/fat components. ${ }^{38,39,41}$ Most of the prototype NEs (primary or Chito-coated platforms) showed good extended stability (at $4^{\circ} \mathrm{C}$ ), evident through minimal change in their mean droplet size (ranging from $\mathrm{D}_{0} \approx 120 \mathrm{~nm}$ to $\left.\mathrm{D}_{90} \approx 140 \mathrm{~nm}\right)$ and particle charge $(<15 \%$ decrease in average $\zeta$-potential over 90 days). Starting after 2 months of cold storage, the particle size of Chito-coated NE trended upwards, paralleled with a gradual decrease in corresponding $\zeta$-potentials, most likely due to gradual "detaching" of the Chito polymer overcoat with extended storage periods (Figure 4A). Only Chito-layered NE containing 60/40 wt\% of + SHS15/TPGS surfactant blend demonstrated considerable increase in particle size (average oil droplet hydrodynamic diameter of $\sim 120 \mathrm{~nm}\left[\mathrm{D}_{0}\right]$ vs $\sim 140$ $\left.\mathrm{nm}\left[\mathrm{D}_{90}\right]\right)$, associated with about $45 \%$ reduction in average surface charge (average $\zeta$-potential of $\sim+20.5 \mathrm{mV}\left[\mathrm{D}_{0}\right]$ vs $\left.\sim+11.9 \mathrm{mV}\left[\mathrm{D}_{90}\right]\right)$ over the same test period (Figure $4 \mathrm{~A}$ ). Physicochemical analyses of similar multilayered emulsions suggest the reduction of sample $\mathrm{pH}$ as one strategy to better stabilize the cationic Chito interfacial layer. ${ }^{27,39}$

In an attempt to determine the consequences of the dehydration (ie, freeze-drying) step during the formation of buccal tablets, the lyophilized oily gel was mildly reconstituted in
A

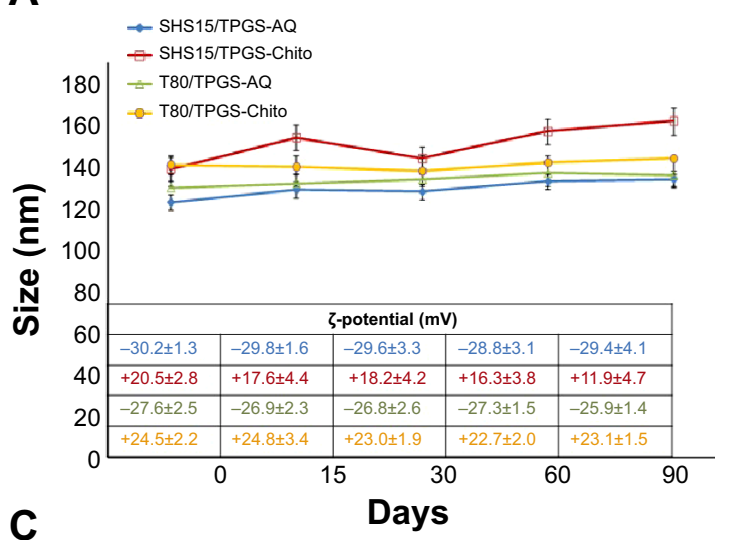

C

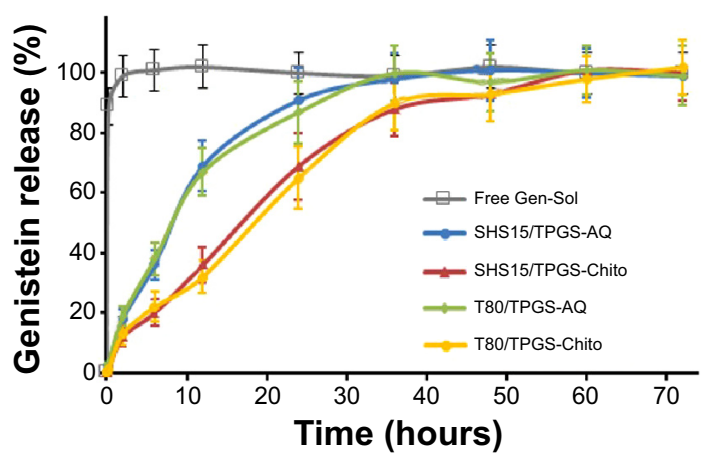

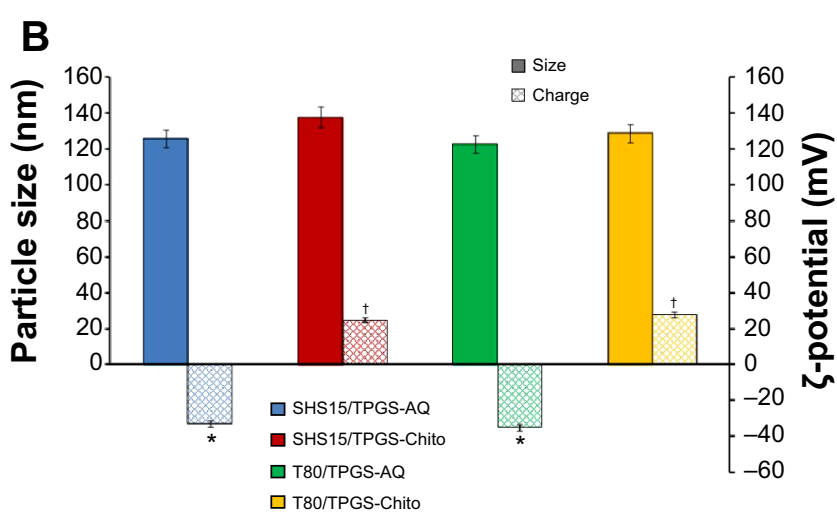

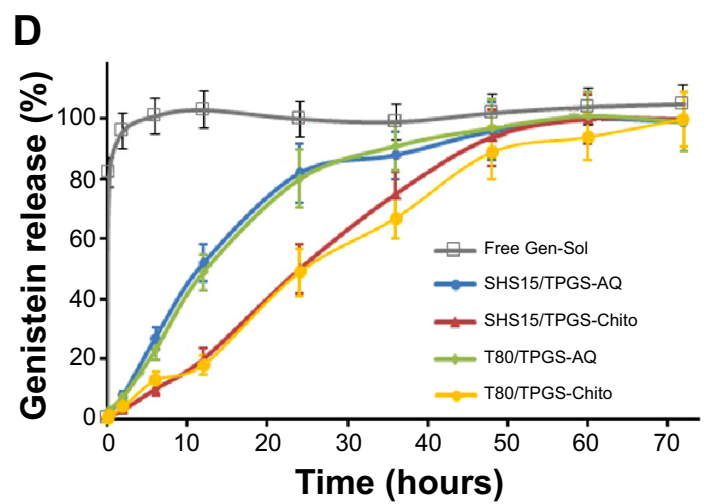

Figure 4 Physicochemical characterization of model layered genistein-NE buccal formulations.

Notes: (A) Stability results based on average particle size and electrical charge of selected layered NE liquid platforms, determined over the period of 90 days. (B) Postreconstitution measurements of both droplet size and surface charge, for lyophilized prototype-layered NE liquid formulations. In vitro release profile of lead Genloaded layered buccal NE formulations, as $(\mathbf{C})$ liquid spray platform and (D) pressed into solid tablet form. Values are shown with \pm SD. Mean values with unlike superscripts $(*, \dagger)$ are statistically different. $(P \leq 0.05) . n=5-7$.

Abbreviations: AQ, aqueous; Chito, chitosan; Gen, genistein; NE, nanoemulsion; SD, standard deviation; Sol, solution; SHSI5, Solutol ${ }^{\circledR}$ HS-I5; T80, Tween ${ }^{\circledR} 80$; TPGS, tocopheryl polyethylene glycol succinate. 
simulated saliva (Figure 4B). For this test, inert tablet excipients - namely, dextrose and MCC - were not yet added to the rehydrated test NE concentrates. The aim was to avoid potential interference of such "wall material", which will ultimately surround the NE particles within the compressed lozenges, upon reconstitution in simulated salivary fluid. Size analysis results indicated successful reformation of droplets, with only 1.3- to 1.8-fold increase in average diameter, for all formulations. Both primary and Chito-layered NE versions deflocculated almost instantaneously with ease, without signs of clumping, showing superior homogeneity, as indicated with very narrow PDI values $(\sim 0.17 \pm 0.05)$. Unexpectedly, the $\zeta$-potential profile of reconstituted NEs increased by almost 1.3 and 1.7 times in amplitude for primary (up to $-38 \mathrm{mV}$ ), and Chito-layered (up to $+26.5 \mathrm{mV}$ ) NEs, respectively. Removal of the external water phase, leading to denser packing of charged surfactant molecules or polymers at the surface of oil droplets, is one plausible explanation.

Another major advantage of layered emulsion systems is the superior control over the release rate of functional agents, based on tuning the thickness and properties of the interfacial polyelectrolyte layer. ${ }^{27,38,41}$ Alternatively, the permeability of the interfacial layer can also be altered in response to surrounding conditions, ${ }^{26}$ such as $\mathrm{pH}$, dilution or temperature. ${ }^{27}$ As anticipated, ${ }^{27}$ the Chito layering of NE particles, whether in liquid or in compressed tablet form (Figure 4C and D), resulted in a significantly slower and more extended release profile of Gen ( $P \geq 0.05$, at $t=6-36$ hours) compared with their primary NE counterparts.

Figure 4C, shows the in vitro Gen-release profile from the native liquid NE platforms as well as from the compressed buccal NE tablet platforms (Figure 4D), comparing primary and Chito-coated NEs of screened formulation (T80:TPGS and SHS15:TPGS, in select 60:40 wt ratio). Overall, Gen-release from the Chito-coated NE-based formulations extended significantly longer ( $\geq 85 \%$ after 48 hours) than that achieved by their primary counterparts ( $>80 \%$ released between $48-36$ hours). Furthermore, the solid tablet platform of Chito-coated NEs (both containing SHS15/TPGS and those containing T80/TPGS) initially displayed significantly greater delayed release of Gen ( $<20 \%$ released by 6 hours) in comparison with their primary Chito-free analogues The evident extended Genrelease profile of Chito-coated NE buccal tablets is (Figure 4D) most likely due to initial formation of a Chito hydrogel overcoat, possibly alongside slower diffusion dynamics of simulated saliva, early on, through the compressed inner matrix of these lozenges.

\section{Ex vivo mucoadhesive assessment of Chito-coated nanoemulsion dosage forms}

The mucoadhesion force measurements (Figure 5A) clearly indicate the significant enhancement of tissue mucoadhesion achieved through Chito-coating of nanodroplets $(P \geq 0.01)$ vs corresponding primary NE-based platforms. Nonetheless, as the Chito-coated NE buccal tablet dosage forms exhibited superior mucoadhesion compared with primary NE-based equivalents, there was no significant difference between formulations that contained T80 vs SHS15, in terms of mucoadhesive forces produced. The absence of appreciable difference between all primary NE-based formulations (ie, containing T80/TPGS vs HS15/TPGS) - whether in liquid or solid dosage form - combined with the lack of Gen-inclusion effect, signifies the dominant role of Chito layering in producing such intense mucoadhesive forces. As a direct outcome of the far superior mucoadhesive forces rendered by our Chito-coated NE buccal tablets, their corresponding tissue residence times were significantly longer ( $t \approx 86-107$ minutes) $(P \leq 0.005)$ than that of any of the Chito-free controls and placebo (Figure 5B). Tissue association times measured for T80-containing tablets were quite close to those measured for the SHS15 tablet control versions (approximately 16-19 minutes). The numerical data were well reflected in the corresponding tissue adhesion assay images of Genloaded lozenges (Figure 5C). Visual inspection of wetted Chito-coated NE-tablets, which were "sandwiched" between excised oral mucosal tissues, revealed apparent swelling and hydrogel adhesion to the buccal mucosal tissues (Figure 5C). Conversely, all other tablets - lacking Chito in their composition - appeared as disintegrated wet powder pieces and separated clumping particles, with minimal tissue adhesion (Figure 5C).

To directly evaluate the physical properties of our proof-of-concept compositions for solid oral tablet/lozenge dosage form, common inactive additives and excipients, such as binders, lubricants, disintegrants, and sugar coatings, were kept minimal, to eliminate their potential effect on the in vitro behavior of our prototype solid formulations. Consequently, physical parameters of oral tablet solidity recorded as relatively low in comparison with typical pharmaceutical industry standards of finished solid dosage forms (Table 2).

Furthermore, while the ranges of tablet hardness were acceptable in general (Table 2), owing to the relatively modest compression forces available (single punch press of 1.5 tons maximum vs typical industrial tableting pressures of 3.0 tons), there was virtually no difference in hardness between all 
A

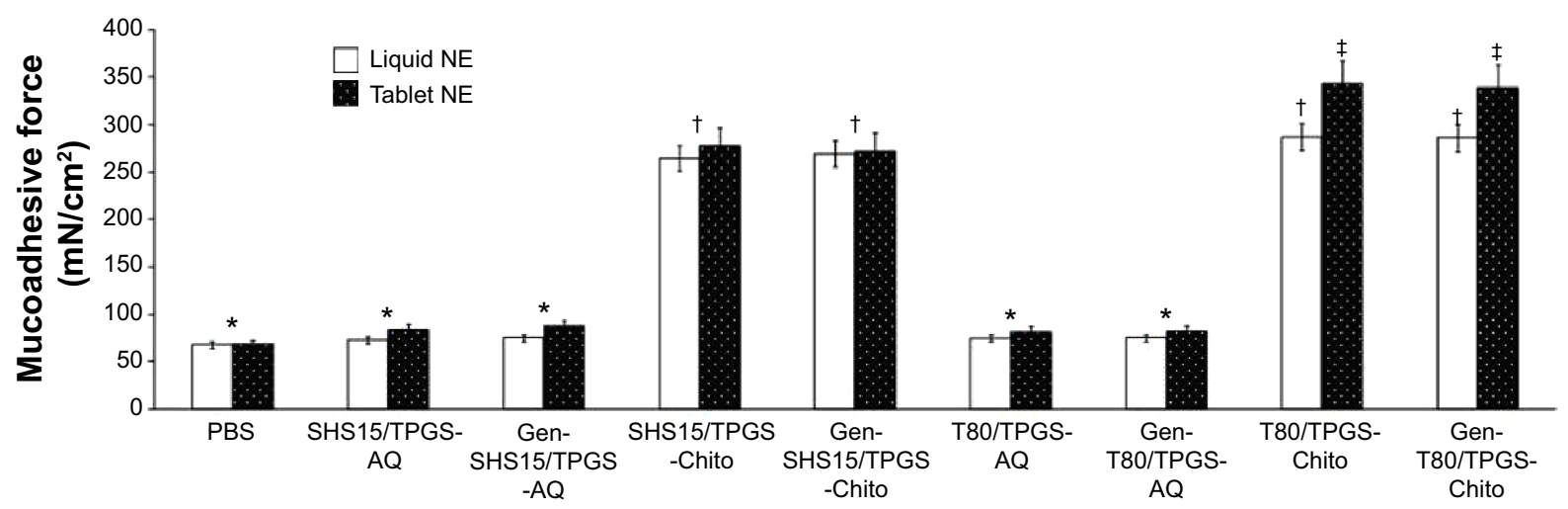

B

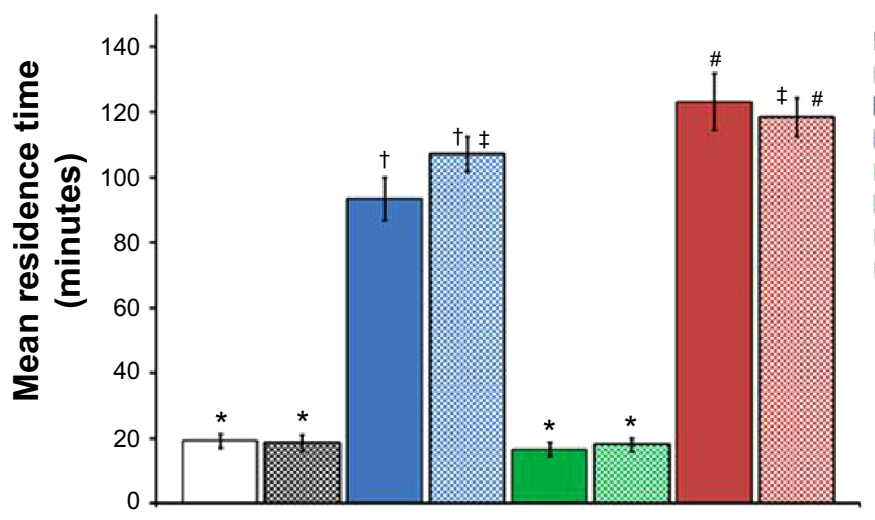

C

$\square$ SHS15/TPGS-AQ

ه Gen-SHS15/TPGS-AQ

$\square$ SHS15/TPGS-Chito

⿴囗大 Gen-SHS15/TPGS-Chito

$\square$ T80/TPGS-AQ

Gen-T80/TPGS-AQ

$\square$ T80/TPGS-Chito

圆 Gen-T80/TPGS-Chito

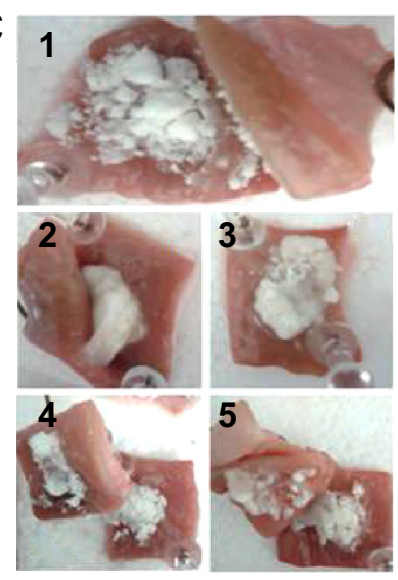

Figure 5 Physical characterization of prototype mucoadhesive Gen-loaded NE solid buccal tablets.

Notes: (A) Ex vivo mucoadhesive force data, measured for both liquid and solid tablet dosage forms, either drug-free or Gen-incorporated into primary(AQ) or layered (Chito) NEs. (B) Ex vivo mucoadhesion residence time measured using a USP 2 dissolution apparatus, for both empty or Gen-loaded NE buccal tablets. (C) Qualitative photographs showing the physical appearance of selected primary (AQ) and Chito-layered NE-based tablets, immediately following the mucoadhesive force testing. CI, PBS (MCC+dextrose) Placebo; C2, Gen-T80/TPGS-Chito; C3, Gen-SHSI5/TPGS-Chito; C4, Gen-T80/TPGS-AQ; C5, Gen-SHSI5/TPGS-AQ. Values are shown with \pm SD. Mean values with unlike superscripts $(*, \#, \neq, \dagger)$ are statistically different $(P \leq 0.05) . n=5-6$.

Abbreviations: AQ, aqueous; Chito, chitosan; Gen, genistein; NE, nanoemulsion; PBS, phosphate-buffered saline; SD, standard deviation; SHSI5, Solutol ${ }^{\circledR}$ HS-I5; T80, Tween $^{\circledR}$ 80; TPGS, tocopheryl polyethylene glycol succinate; USP 2, United States Pharmacopiea type-2.

Table 2 Physical properties of prototype solid dosage forms: Gen-loaded and placebo NE-based buccal tablets

\begin{tabular}{|c|c|c|c|c|c|c|}
\hline $\begin{array}{l}\text { NE } \\
\text { (surfactant mix, wt\%) }\end{array}$ & Chitosan & $\begin{array}{l}\text { Mean } \\
\text { weight }(\mathbf{m g})\end{array}$ & $\begin{array}{l}\text { Mean hardness } \\
\left(\mathrm{N} / \mathrm{cm}^{2}\right)\end{array}$ & Friability (\%) & $\begin{array}{l}\text { Mean } \\
\text { surface pH }\end{array}$ & $\begin{array}{l}\text { Mean swelling } \\
\text { index (post-4 hr) }\end{array}$ \\
\hline \multirow[t]{2}{*}{ SHSI5 (100) } & + & $149 \pm 1.3$ & $2.17 \pm 0.1$ & $0.7 I \pm 0.02$ & $5.78 \pm 0.05$ & $78.2 \pm 8.5^{*}$ \\
\hline & - & $148 \pm 0.8$ & $2.21 \pm 0.18$ & $0.75 \pm 0.05$ & $6.27 \pm 0.04$ & $35.4 \pm 4.8^{\dagger}$ \\
\hline \multirow[t]{2}{*}{ SHSI5:TPGS (60:40) } & + & $150 \pm 0.4$ & $2.61 \pm 0.06$ & $0.68 \pm 0.04$ & $5.83 \pm 0.06$ & $87.2 \pm 7.7^{*}$ \\
\hline & - & $149 \pm 0.7$ & $2.59 \pm 0.07$ & $0.72 \pm 0.06$ & $6.19 \pm 0.03$ & $42.2 \pm 3.5^{\dagger}$ \\
\hline \multirow[t]{2}{*}{ SHSI5:TPGS (40:60) } & + & $149 \pm 0.9$ & $2.53 \pm 0.05$ & $0.74 \pm 0.0 \mathrm{I}$ & $5.73 \pm 0.04$ & $86.5 \pm 5.8^{*}$ \\
\hline & - & $\mid 48 \pm 1.2$ & $2.45 \pm 0.06$ & $0.76 \pm 0.02$ & $6.31 \pm 0.04$ & $38.7 \pm 6 . I^{\dagger}$ \\
\hline \multirow[t]{2}{*}{ T80 (100) } & + & $149 \pm 1.1$ & $2.18 \pm 0.08$ & $0.67 \pm 0.03$ & $5.8 I \pm 0.02$ & $79.4 \pm 8.2 *$ \\
\hline & - & $150 \pm 0.6$ & $1.98 \pm 0.13$ & $0.7 I \pm 0.02$ & $6.19 \pm 0.06$ & $40.1 \pm 3.1^{\dagger}$ \\
\hline \multirow[t]{2}{*}{ T80:TPGS (60:40) } & + & $149 \pm 1.6$ & $2.5 \pm 0.09$ & $0.7 I \pm 0.05$ & $5.76 \pm 0.05$ & $88.8 \pm 3.9 *$ \\
\hline & - & $149 \pm 1.2$ & $2.42 \pm 0.07$ & $0.73 \pm 0.04$ & $6.22 \pm 0.02$ & $40.2 \pm 5.2^{\dagger}$ \\
\hline \multirow[t]{2}{*}{ T80:TPGS (40:60) } & + & $149 \pm 0.9$ & $2.39 \pm 0.12$ & $0.7 \pm 0.03$ & $5.79 \pm 0.0$ & $87.9 \pm 6.5^{*}$ \\
\hline & - & $150 \pm 0.6$ & $2.3 I \pm 0.05$ & $0.75 \pm 0.04$ & $6.21 \pm 0.03$ & $41.0 \pm 3.6^{\dagger}$ \\
\hline \multirow[t]{2}{*}{ TPGS $(100)$} & + & $149 \pm 0.5$ & $2.5 I \pm 0.09$ & $0.75 \pm 0.02$ & $5.73 \pm 0.05$ & $89.1 \pm 6.6 *$ \\
\hline & - & $148 \pm 0.8$ & $2.35 \pm 0.1$ & $0.78 \pm 0.06$ & $6.25 \pm 0.02$ & $39.3 \pm 4.0^{\dagger}$ \\
\hline
\end{tabular}

Notes: Values are expressed as mean $( \pm S D)$. Mean values with unlike superscripts $(*, \dagger)$ are statistically different $(P \leq 0.05)$. $n=6-10$.

Abbreviations: $D_{60}$, post-60 days measurement; Gen, genistein; NE, nanoemulsion; SD, standard deviation; SHSI5, Solutol ${ }^{\circledR}$ HS; T80, Tween ${ }^{\circledR} 80$; TPGS, tocopheryl polyethylene glycol succinate. 
tested NE-based tablet formulations. Standing rather above average, the SHS15 (SHS15:TPGS at 40:60 wt\%) NE-based tablets appeared to be comparatively the hardest tablet composition $(0.1>P>0.05)$, whether they contained primary NE or mucoadhesive NE components. Consequently, the overall friability of our proof-of-principle buccal tablets was also on the low side (range between $0.7 \%-0.8 \%$ ), with marginally better values obtained with the inclusion of Chito-coating as an NE component (Table 2). As expected, upon sufficient hydration, Chito-layered NEs produced marked hydrogelinduced swelling throughout the matrix and surface of their buccal tablets. Starting after 20 minutes of hydration, the mucoadhesive buccal tablets swelled to at least twice the size of their Chito-free counterparts (as measured after 4 hours), showing an apparent hydrogel overcoat surrounding each tablet. The mean surface $\mathrm{pH}$ of mucoadhesive tablets was slightly lower (average $\mathrm{pH} \sim 5.8 \pm 0.2$ ) than the corresponding tablet controls ( $\mathrm{pH}$ of Chito-free tablets $\sim 6.1 \pm 0.2$ ), possibly due to the progressive swelling of Chito-hydrogel at the surface.

\section{Cell-based assays of Gen-mucoadhesive nanoplatforms}

Although no appreciable difference was found between liquid NE based on SHS15 or T80, in physicochemical properties as well as mucoadhesion test results, their corresponding buccal tablet forms were examined side by side. These tablets (either as primary or Chito-coated NEs) were virtually equivalent in physical tests. Yet both tissue mucoadhesion assays of force and residence time proved the advantage of the Chito-coated T80/TPGS formulation buccal tablets. Moreover, both primary/AQ NE and Chito-coated NE tablets with T80/TPGS successfully passed our L929 cell culture-based biocompatibility safety assessment (Figure 6A and B), which was derived from International Organization for Standardization (ISO)-10993/Food and Drug Administration (FDA) draft guidance for submicron and nanotechnology components. ${ }^{42}$ Moreover, based on the time-dependent biocompatibility screen of nanoemulsified platforms, both primary and Chito-layered T80 NEs showed slightly milder impact on test L929 (human connective tissue) cell vitality relative to the corresponding SHS15 NE analogues (Figure 6), more visibly in micrographs (Figure 6B) starting after 24 hours of coincubation (estimated viability values were $74 \% \pm 3.6 \%$ and $79 \% \pm 3.9 \%$ vs $68 \% \pm 3.4 \%$ and $72 \% \pm 4.2 \%$, respectively) (Figure $6 \mathrm{~A}$ ).

Therefore, NE platforms based on the T80 surfactant blend were rationally selected as the prototype formulation for further cell-based antiproliferative assays, in order to verify the anticancer effectiveness of our Gen-loaded buccal formulations against two different oral carcinoma cell lines (Figure 7). The concentration-dependent cytotoxicity profiles revealed no appreciable activity for the Gen-free NE controls against oral carcinoma cells. In contrast, data obtained from human tongue squamous cell carcinoma culture, SCC-4 (Figure 7A), revealed evident antiproliferative activity of both the AQ T80/TPGS (50\% inhibition level $\left[\mathrm{IC}_{50}\right]=0.64 \pm 0.15$ $\mathrm{mM})$ and Chito-coated T80/TPGS $\left(\mathrm{IC}_{50}=0.62 \pm 0.11\right.$ $\mathrm{mM}$ ) Gen-loaded formulations. In similar fashion, these
A

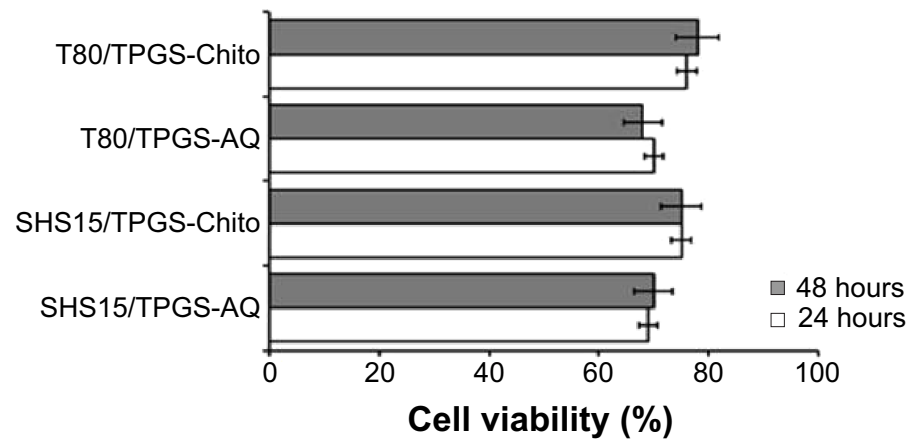

B

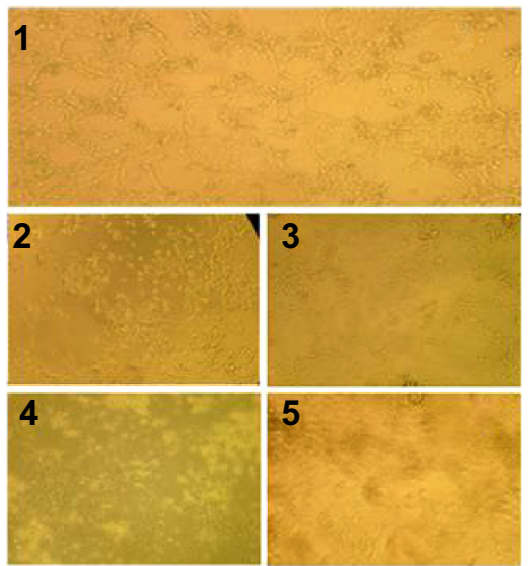

Figure 6 Biocompatibility assay of primary and layered NEs.

Notes: Cell viability assay for lead Chito-layered NE formulations, compared with primary aqueous NEs - employing untreated culture media as negative control - all coincubated with (A) murine areolar fibroblast (L929) cells. Values are shown with \pm SE. $n=4-5$. (B) Qualitative transmitted light micrographs demonstrating the morphology of unstained L929 cells, following 24-hour coincubation with various treatments: (I) negative control serum-free EMEM medium, (2) T80/TPGS-Chito NE, (3) T80/TPGS-AQ NE, (4) SHSI5/TPGS-Chito NE, and (5) SHSI5/TPGS-AQ NE. The selected liquid NEs were added to confluent cells (as two hundred fold dilutions) in serum-free EMEM medium. Abbreviations: AQ, aqueous; EMEM, Eagle's minimal essential medium; NE, nanoemulsion; SE, standard error; SHSI5, Solutol ${ }^{\circledR}$ HS- I5; T80, Tween ${ }^{\circledR} 80$; TPGS, tocopheryl polyethylene glycol succinate. 
A

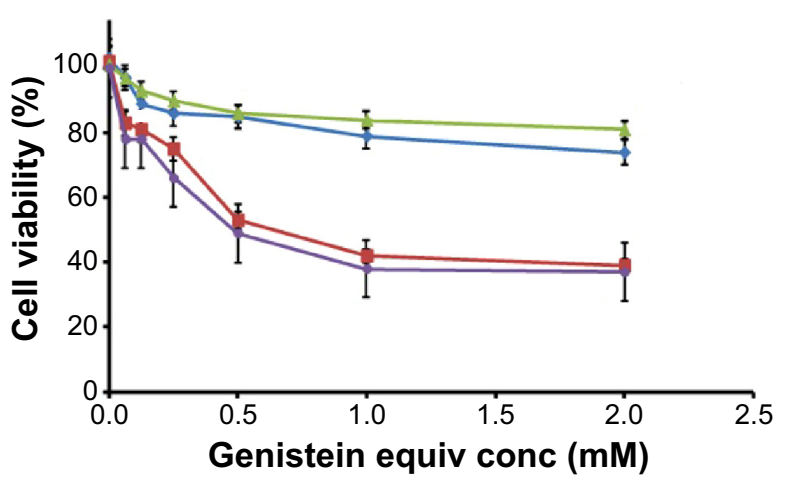

B

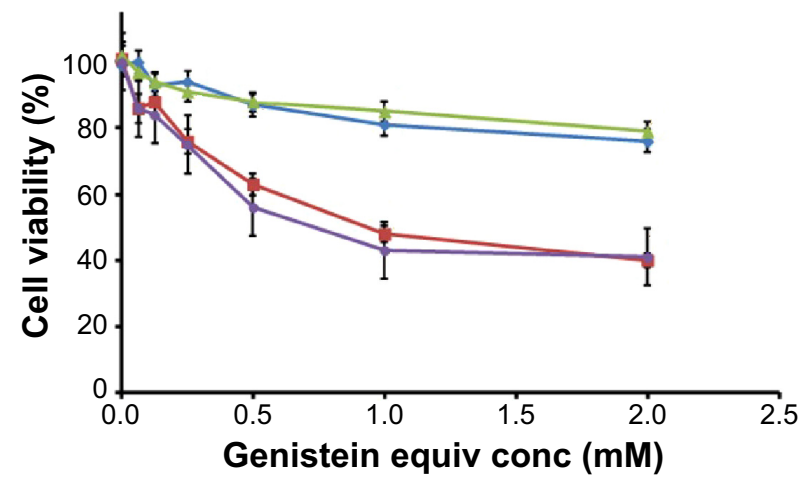

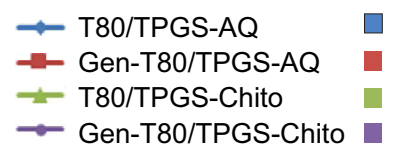

C

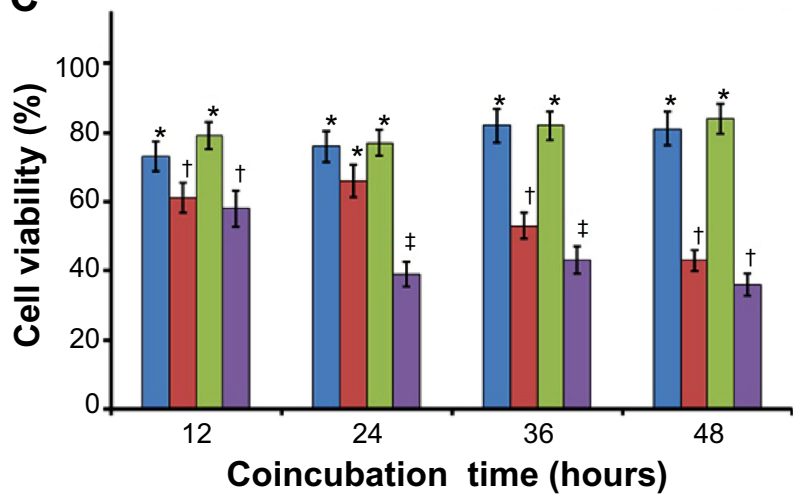

D

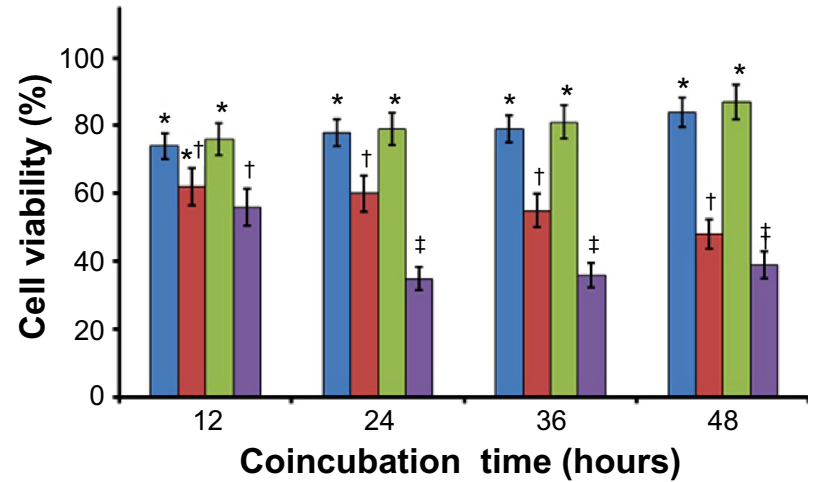

Figure 7 In vitro anticancer activity of prototype Gen-layered NEs against oral carcinomas.

Notes: Concentration-dependent cytotoxicity profiles of different Gen-loaded cationic/ Chito-layered NEs compared with corresponding primary (AQ) NE controls, in (A) FaDu (pharyngeal) and (B) SCC-4 (tongue, human squamous cell carcinoma) cells. Plus, temporal cytotoxicity profile (over 48 hours of coincubation, at I mM equivalent Gen concentration), in test oral cancer cell lines, $\mathrm{FaDu}(\mathbf{C})$ and SCC-4 (D). Mean values with unlike superscripts $(*, \neq, \dagger)$ are statistically different $(P \leq 0.05)$. $n=4-6$.

Abbreviations: AQ, aqueous; Chito, chitosan; equiv conc, equivalent concentration; Gen, genistein; NE, nanoemulsion; SHSI5, Solutol ${ }^{\circledR}$ HS-15; T80, Tween ${ }^{\circledR} 80 ;$ TPGS, tocopheryl polyethylene glycol succinate.

Gen-containing NE formulations, both AQ and Chitocoated, containing T80/TPGS manifested marked anticancer activity against $\mathrm{FaDu}$ human pharyngeal squamous cell carcinoma $\left(\mathrm{IC}_{50}=0.88 \pm 0.14\right.$ and $0.87 \pm 0.18 \mathrm{mM}$, respectively) (Figure 7B). That is to say, after 48 hours of coincubation, Gen-incorporating prototype mucoadhesive NE proved as effective as its primary NE analogues against in vitro test oral cancer models. Nevertheless, in both cancer cell cultures, the temporal cytotoxicity assays (Figure 7C and D) revealed a significantly stronger anticancer profile of the cationic Gen-loaded Chito-layered NE compared with the corresponding primary NE treatment $(P \leq 0.05)$, specifically at earlier incubation times (24-36 hours). Only after 48 hours of coincubation did the primary/AQ NE formulation of Gen reduce cancer cell viability closer to levels produced by its Chitocoated NE analogues. Effectively, the lead mucoadhesive buccal NE formulation of Gen manifested strong in vitro cytotoxicity, in concentration-dependent and time-dependent manners, compared with its Chito-free analogues. Most importantly, such early superior in vitro cytotoxicity of Chito-layered NE, noted by the 24-hour time point, can be attributed to fast electrostatic adhesion of these cationic NE droplets to oral cancer cell membranes. Hence, despite their somewhat slower Gen-release profile ( $<36$ hours compared with their primary NE controls), these Chito-layered NEs have the advantage of releasing their drug cargo at very close proximity to target cancer cells, efficiently killing them. Eventually, both Gen-containing NE formulations appear to have reached equivalent cancer cell kill ratios - by 48 hours, in both cancer cell models.

\section{Conclusion}

The ability to rationally assess and control interfacial properties of our Chito-layered nanoemulsion systems permitted 
superior drug-loaded droplet designs, with improved stability and added mucoadhesive functional performance. Further development of select proof-of-concept buccal liquids and tablets resulted in sustained delivery of the antiproliferative drug candidate, Gen, yielding distinct anticancer effects. Encouraging data from our new mucoadhesive buccal sprays and lozenges suggest the potential of such platforms to be used as adjuvant therapy for oral cancer patients. A chief aim would be to eliminate any residual cancerous cells remaining after tumor removal, hence preventing postsurgical tumor recurrence or risk of postoperative metastasis of residual detached cancerous cells.

\section{Acknowledgments}

This research was partially funded by a Midwestern University Dental School Research Student Stipend award for A Gavin.

\section{Disclosure}

The authors report no conflicts of interest in this work.

\section{References}

1. van der Tol IG, de Visscher JG, Jovanovic A, van der Waal I. Risk of second primary cancer following treatment of squamous cell carcinoma of the lower lip. Oral Oncol. 1999;35(6):571-574.

2. Koch WM, Stafford E, Bajaj G. Cancer of the oral cavity: General principles and management. In: Harrison LB, Sessions RB, Hong WK, editors. Head and Neck Cancer: A Multidisciplinary Approach. 3rd ed. Philadelphia, PA: Lippincott Williams \& Wilkins; 2009:250-264.

3. Po Wing Yuen A, Lam KY, Lam LK, et al. Prognostic factors of clinically stage I and II oral tongue carcinoma-A comparative study of stage, thickness, shape, growth pattern, invasive front malignancy grading, Martinez-Gimeno score, and pathologic features. Head Neck. 2002; 24(6):513-520.

4. Harrison LB, Sessions RB, Hong WK. Head and Neck Cancer: A Multidisciplinary Approach. 3rd ed. Philadelphia, PA: Lippincott Williams \& Wilkins; 2009.

5. Smart JD. Recent developments in the use of bioadhesive systems for delivery of drugs to the oral cavity. Crit Rev Ther Drug Carrier Syst. 2004;21(4):319-344.

6. Paderni C, Compilato D, Giannola LI, Campisi G. Oral local drug delivery and new perspectives in oral drug formulation. Oral Surg Oral Med Oral Pathol Oral Radiol. 2012;114(3):e25-e34.

7. Mizrahi B, Domb AJ. Mucoadhesive polymers for delivery of drugs to the oral cavity. Recent Pat Drug Deliv Formul. 2008;2(2):108-119.

8. Shaikh R, Raj Singh TR, Garland MJ, Woolfson AD, Donnelly RF. Mucoadhesive drug delivery systems. J Pharm Bioallied Sci. 2011;3(1): 89-100.

9. Salamat-Miller N, Chittchang M, Johnston TP. The use of mucoadhesive polymers in buccal drug delivery. Adv Drug Deliv Rev. 2005;57(1): 1666-1691.

10. Shojaei AH, Berner B, Xiaoling L. Transbuccal delivery of acyclovir: I. In vitro determination of routes of buccal transport. Pharm Res. 1998; 15(8):1182-1188.

11. Banerjee S, Li Y, Wang Z, Sarkar FH. Multi-targeted therapy of cancer by genistein. Cancer Lett. 2008;269(2):226-242.

12. Spinozzi F, Pagliacci MC, Migliorati G, et al. The natural tyrosine kinase inhibitor genistein produces cell cycle arrest and apoptosis in Jurkat T-leukemia cells. Leuk Res. 1994;18(6):431-439.
13. Lian F, Li Y, Bhuiyan M, Sarkar FH. p53-independent apoptosis induced by genistein in lung cancer cells. Nutr Cancer. 1999;33(2):125-131.

14. Baxa DM, Luo X, Yoshimura FK. Genistein induces apoptosis in T lymphoma cells via mitochondrial damage. Nutr Cancer. 2005;51(1): 93-101.

15. Li Y, Ahmed F, Ali S, Philip PA, Kucuk O, Sarkar FH. Inactivation of nuclear factor kappaB by soy isoflavone genistein contributes to increased apoptosis induced by chemotherapeutic agents in human cancer cells. Cancer Res. 2005;65(15):6934-6942.

16. Messina S, Bitto A, Aguennouz M, et al. The soy isoflavone genistein blunts nuclear factor kappa-B, MAPKs and TNF- $\alpha$ activation and ameliorates muscle function and morphology in mdx mice. Neuromuscul Disord. 2011;21(8):579-589.

17. Yoshimura FK. Genistein induces apoptosis via mitochondrial damage in acute lymphoblastic leukemia T-cell lines. Cancer Ther. 2004;2: 429-440.

18. Phan V, Walters J, Brownlow B, Elbayoumi T. Enhanced cytotoxicity of optimized liposomal genistein via specific induction of apoptosis in breast, ovarian and prostate carcinomas. J Drug Target. 2013; 21(10):1001-1011.

19. Pham J, Brownlow B, Elbayoumi T. Mitochondria-specific proapoptotic activity of genistein lipidic nanocarriers. Mol Pharm. 2013;10(10):3789-3800.

20. Wu JG, Ge J, Zhang YP, Yu Y, Zhang XY. Solubility of genistein in water, methanol, ethanol, propan-2-ol, 1-butanol, and ethyl acetate from (280 to 333) K. J Chem Eng Data. 2010;55(11):5286-5288.

21. Tang J, Xu N, Ji H, Liu H, Wang Z, Wu L. Eudragit nanoparticles containing genistein: formulation, development, and bioavailability assessment. Int J Nanomedicine. 2011;6:2429-2435.

22. de Vargas BA, Bidone J, Oliveira LK, Koester LS, Bassani VL, Teixeira HF. Development of topical hydrogels containing genisteinloaded nanoemulsions. J Biomed Nanotechnol. 2012;8(2):330-336.

23. Kwon SH, Kim SY, Ha KW, et al. Pharmaceutical evaluation of genistein-loaded pluronic micelles for oral delivery. Arch Pharm Res. 2007;30(9):1138-1143.

24. Kitagawa S, Inoue K, Teraoka R, Morita SY. Enhanced skin delivery of genistein and other two isoflavones by microemulsion and prevention against UV irradiation-induced erythema formation. Chem Pharm Bull (Tokyo). 2010;58(3):398-401.

25. Jordan M, Nayel A, Brownlow B, Elbayoumi T. Development and evaluation of tocopherol-rich argan oil-based nanoemulsions as vehicles possessing anticancer activity. J Biomed Nanotechnol. 2012;8(6):944-956.

26. McClements DJ, Decker EA, Weiss J. Emulsion-based delivery systems for lipophilic bioactive components. J Food Sci. 2007;72(8): R109-R124.

27. Guzey D, McClements DJ. Formation, stability and properties of multilayer emulsions for application in the food industry. Adv Colloid Interface Sci. 2006;128-130:227-248.

28. Faulk A, Weissig V, Elbayoumi T. Mitochondria-specific nano-emulsified therapy for myocardial protection against doxorubicin-induced cardiotoxicity. Methods Mol Biol. 2013;991:99-112.

29. Berth G, Voigt A, Dautzenberg H, Donath E, Möhwald H. Polyelectrolyte complexes and layer-by-layer capsules from chitosan/ chitosan sulfate. Biomacromolecules. 2002;3(3):579-590.

30. Shirsand S, Suresh S, Keshavshetti G, Swamy P, Reddy PV. Formulation and optimization of mucoadhesive bilayer buccal tablets of atenolol using simplex design method. Int J Pharm Investig. 2012;2(1): 34-41.

31. Charde S, Mudgal M, Kumar L, Saha R. Development and evaluation of buccoadhesive controlled release tablets of lercanidipine. AAPS Pharm Sci Tech. 2008;9(1):182-190.

32. Desai KG, Kumar TM. Preparation and evaluation of a novel buccal adhesive system. AAPS Pharm Sci Tech. 2004;5(3):e35.

33. Shidhaye SS, Saindane NS, Sutar S, Kadam V. Mucoadhesive bilayered patches for administration of sumatriptan succinate. AAPS Pharm Sci Tech. 2008;9(3):909-916. 
34. Lvov YM, Pattekari P, Zhang X, Torchilin V. Converting poorly soluble materials into stable aqueous nanocolloids. Langmuir. 2011;27(3): 1212-1217.

35. Espada CE, Berra MA, Martinez MJ, Eynard AR, Pasqualini ME. Effect of Chia oil (Salvia Hispanica) rich in omega-3 fatty acids on the eicosanoid release, apoptosis and T-lymphocyte tumor infiltration in a murine mammary gland adenocarcinoma. Prostaglandins Leukot Essent Fatty Acids. 2007;77(1):21-28.

36. Ayerza R. The seed's protein and oil content, fatty acid composition, and growing cycle length of a single genotype of chia (Salvia hispanica L.) as affected by environmental factors. JOleo Sci. 2009;58(7): 347-354.

37. Comba A, Maestri DM, Berra MA, et al. Effect of $\omega-3$ and $\omega-9$ fatty acid rich oils on lipoxygenases and cyclooxygenases enzymes and on the growth of a mammary adenocarcinoma model. Lipids Health Dis. 2010;9:112.

38. Ogawa S, Decker EA, McClements DJ. Production and characterization of $\mathrm{O} / \mathrm{W}$ emulsions containing cationic droplets stabilized by lecithinchitosan membranes. J Agric Food Chem. 2003;51(9):2806-2812.
39. Shaw LA, McClements DJ, Decker EA. Spray-dried multilayered emulsions as a delivery method for omega-3 fatty acids into food systems. J Agric Food Chem. 2007;55(8):3112-3119.

40. Surh J, Gu YS, Decker EA, McClements DJ. Influence of environmental stresses on stability of $\mathrm{O} / \mathrm{W}$ emulsions containing cationic droplets stabilized by SDS-fish gelatin membranes. J Agric Food Chem. 2005; 53(10):4236-4244.

41. Payet L, Terentjev EM. Emulsification and stabilization mechanisms of $\mathrm{o} / \mathrm{w}$ emulsions in the presence of chitosan. Langmuir. 2008;24(21):12247-12252.

42. Battig A, Hiebl B, Feng Y, Lendlein A, Behl M. Biological evaluation of degradable, stimuli-sensitive multiblock copolymers having polydepsipeptide- and poly( $\varepsilon$-caprolactone) segments in vitro. Clin Hemorheol Microcirc. 2011;48(1):161-172.
International Journal of Nanomedicine

\section{Publish your work in this journal}

The International Journal of Nanomedicine is an international, peerreviewed journal focusing on the application of nanotechnology in diagnostics, therapeutics, and drug delivery systems throughout the biomedical field. This journal is indexed on PubMed Central, MedLine, CAS, SciSearch ${ }^{\circledR}$, Current Contents ${ }^{\circledR} /$ Clinical Medicine,

\section{Dovepress}

Journal Citation Reports/Science Edition, EMBase, Scopus and the Elsevier Bibliographic databases. The manuscript management system is completely online and includes a very quick and fair peer-review system, which is all easy to use. Visit http://www.dovepress.com/ testimonials.php to read real quotes from published authors. 